\title{
Illustrating the Use of Vector Clocks in Property Detection: An Example and a Counter-Example
}

\author{
Michel Raynal \\ IRISA, Campus de Beaulieu, 5042 Rennes Cedex, France \\ raynal@irisa.fr
}

\section{Introduction}

Logical (scalar, vector or matrix) clocks are a powerful mechanism used by a lot of distributed algorithms. This paper is on vector clocks: it surveys their main features and is particularly focused on their power and their limitation. In that sense, this paper complements [5, 6] and may be seen as a critical and practical introduction to vector clocks.

The paper is divided into four main sections. Section 2 introduces a model for distributed executions. Section 3 describes vector clocks and their basic properties. Vector clocks are a simple mechanism that allows processes to track causality between the events they produce. Then, Section 4 and Section 5 study two problems related to causality. The first problem consists in detecting a conjunction of stable local predicates. The second problem consists in recognizing the occurrence of a very simple event pattern. It is shown that simple vector clocks are insufficient to solve the second problem which, actually, requires more sophisticated clocks, namely, vector of vector clocks. So, this paper exhibits a frontier between problems that can be solved using simple vector clocks and problems requiring more sophisticated vector clock systems.

\section{Distributed Computations}

\subsection{Partially Ordered Set of Events}

Distributed Computations A distributed program is made up of $n$ sequential local programs which can communicate and synchronize only by exchanging messages. The execution of a local program gives rise to a sequential process. Let $P_{1}, \ldots, P_{n}$ be this finite set of processes. We assume that, at run-time, each ordered pair of communicating processes $\left(P_{i}, P_{j}\right)$ is connected by a reliable channel. Message transmission delays are finite but unpredictable, process speeds are positive but arbitrary: the underlying computation model is asynchronous. Partial Order of Events Execution of an internal/send/receive statement produces a corresponding internal/send/receive event. Let $e_{i}^{x}$ be the $x$-th event produced by process $P_{i}$. The sequence $h_{i}=e_{i}^{1} e_{i}^{2} \ldots e_{i}^{x} \ldots$ constitutes the history of $P_{i}$. Let $H$ be the set of events produced by a distributed computation. This set is structured as a partial order by Lamport's causal precedence relation [3], denoted " $\rightarrow$ " and defined as follows: 


$$
\begin{aligned}
&(i=j \wedge x \leq y) \text { (local precedence) } \vee \\
&\left.e_{i}^{x} \rightarrow e_{j}^{y} \Leftrightarrow\left(\exists m: e_{i}^{x}=\operatorname{send}(m) \wedge e_{j}^{y}=\text { receive }(m)\right) \text { (msg prec. }\right) \vee \vee \\
&\left(\exists e_{k}^{z}: e_{i}^{x} \rightarrow e_{k}^{z} \wedge e_{k}^{z} \rightarrow e_{j}^{y}\right) \quad(\text { transitive closure) }
\end{aligned}
$$

The partial order $\widehat{H}=(H, \rightarrow)$ constitutes a model of the distributed computation it is associated with. Figure 1 depicts a distributed computation where events are denoted by black points. Two events $e$ and $f$ are concurrent (or causally independent $)$ if $\neg(e \rightarrow f) \wedge \neg(f \rightarrow e)$. The causal past of event $e$ is the partially ordered set of events $f$ such that $f \rightarrow e$. Similarly, the causal future of event $e$ is the partially ordered set of events $f$ such that $e \rightarrow f$.

\subsection{Partially Ordered Set of Local States}

Local States Let $\sigma_{i}^{0}$ be the initial state of process $P_{i}$. Event $e_{i}^{x}$ entails $P_{i}$ 's local state change from $\sigma_{i}^{x-1}$ to $\sigma_{i}^{x}: \sigma_{i}^{x}$ is the local state of $P_{i}$ resulting from its partial history $h_{i}^{x}=e_{i}^{1} \ldots e_{i}^{x}$. We say that $e_{i}^{y}$ belongs to $\sigma_{i}^{x}\left(\operatorname{denoted} e_{i}^{y} \in \sigma_{i}^{x}\right)$ if $y \leq x$. In Figure 1 local states are represented by rectangular boxes.

Partial Order of Local States Let $\Sigma$ be the set of all local states associated with a distributed computation $\widehat{H}$. Lamport's precedence relation can be extended to local states in the following way:

$$
\left(\sigma_{i}^{x} \rightarrow \sigma_{j}^{y}\right) \Leftrightarrow\left(e_{i}^{x+1} \rightarrow e_{j}^{y}\right) \quad(S E)
$$

Local states not related by " $\rightarrow$ " are said to be concurrent, (denoted \|). More formally: $\left(\sigma_{i}^{x} \| \sigma_{j}^{y}\right) \Leftrightarrow\left(\neg\left(\sigma_{i}^{x} \rightarrow \sigma_{j}^{y}\right) \wedge \neg\left(\sigma_{j}^{y} \rightarrow \sigma_{i}^{x}\right)\right)$. In Figure 1, we have $\sigma_{3}^{1} \rightarrow \sigma_{1}^{4}$ and $\sigma_{1}^{3} \| \sigma_{3}^{5}$. As for events, the causal past of a local state $\sigma_{i}^{x}$ is the partially ordered set of local states $\sigma_{k}^{z}$ such that $\sigma_{k}^{z} \rightarrow \sigma_{i}^{x}$.

Consistent Global States A global state $\Sigma$ is a set of $n$ local states $\left(\sigma_{1}, \cdots, \sigma_{n}\right)$ one from each process. It is consistent if $\forall i \neq j$ we have $\sigma_{i} \| \sigma_{j}$. This means that a global state $\Sigma=\left(\sigma_{1}, \cdots, \sigma_{n}\right)$ is consistent if, for any pair of its local states $\left(\sigma_{i}, \sigma_{j}\right)$, there is no message $m$ such that receive $(m) \in \sigma_{i} \wedge \operatorname{send}(m) \notin \sigma_{j}$. If such a message $m$ exists, it is called orphan with respect to the pair $\left(\sigma_{i}, \sigma_{j}\right)$. Let us again consider Figure 1. $\Sigma_{1}=\left(\sigma_{1}^{3}, \sigma_{2}^{4}, \sigma_{3}^{2}\right)$ is consistent, while $\Sigma_{2}=\left(\sigma_{1}^{5}, \sigma_{2}^{6}, \sigma_{3}^{4}\right)$ is not consistent. There is no orphan message with respect to $\Sigma_{1}$, while $m_{4}$ is orphan with respect to the pair $\left(\sigma_{3}^{4}, \sigma_{2}^{6}\right) \subset \Sigma_{2}$. Actually, $m_{4}$ creates the dependency $\sigma_{3}^{4} \rightarrow \sigma_{2}^{6}$ which makes $\Sigma_{2}$ inconsistent.

\section{Vector Clocks}

As a concept, with the associated theory, vector clocks have been introduced in 1988, simultaneously and independently by Fidge [1] and by Mattern [4.

A Causality Tracking Timestamping Mechanism A vector clock system is a mechanism that associates timestamps with events (local states) in such a way that the comparison of their timestamps indicates whether the corresponding events (local states) are or not causally related (and, if they are, which one is the first). This timestamping system is implemented in the following way. Each process $P_{i}$ has a vector of integers $V C_{i}[1 . . n]$ and: 


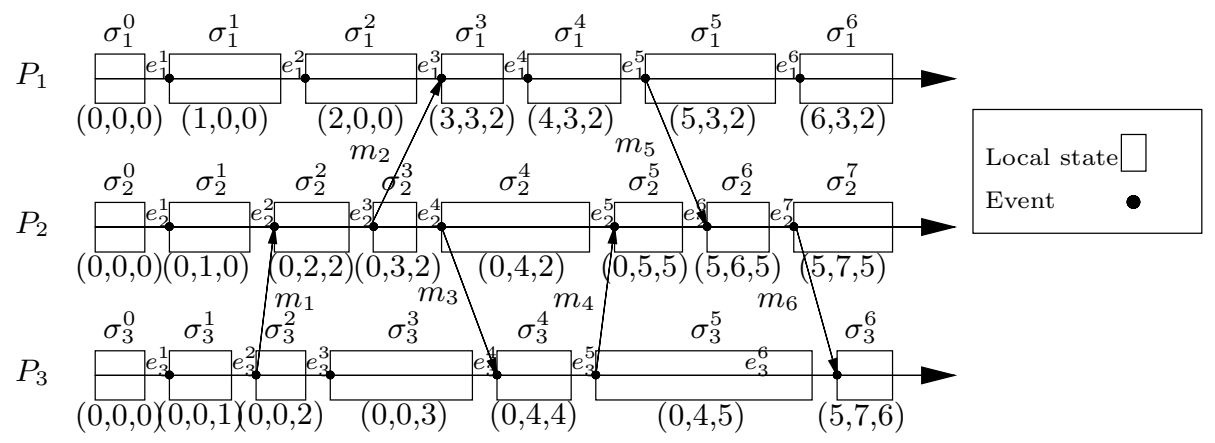

Fig. 1. Local States of a Distributed Computation

R1 Each time it produces an event, $P_{i}$ increments its vector clock entry $V C_{i}[i]$ $\left(V C_{i}[i]:=V C_{i}[i]+1\right)$ to indicate it has progressed.

R2 When a process $P_{i}$ sends a message $m$, it attaches to it the current value of $V C_{i}$. Let $m . V C$ denote this value.

R3 When $P_{i}$ receives a message $m$, it updates its vector clock in the following way: $\forall x: V C_{i}[x]:=\max \left(V C_{i}[x], m . V C[x]\right.$ ) (this operation is usually abbreviated as $\left.V C_{i}:=\max \left(V C_{i}, m . V C\right)\right)$.

Note that $V C_{i}[i]$ counts the number of events produced so far by $P_{i}$. Moreover, $V C_{i}[j]$ represents the number of events produced by $P_{j}$ that belongs to the current causal past of $P_{i}$. When a process $P_{i}$ produces an event $e$ (and enters the associated local state $\sigma$ ) it associates with them a vector timestamp whose value is equal to the current value of $V C_{i}$, namely, e.VC= $\sigma \cdot V C=$ current value of $V C_{i}$. Figure 1 shows vector timestamp values associated with events and local states. As an example we have: $e_{4}^{2} \cdot V C=\sigma_{4}^{2} \cdot V C=(0,4,2)$.

Let $e . V C$ and $f . V C$ be the vector timestamps associated with two distinct events $e$ and $f$, respectively. The following property is the fundamental property associated with vector clocks [1, 2, 4] 1 :

$$
((e \rightarrow f) \Leftrightarrow((\forall k: e . V C[k] \leq f . V C[k]) \wedge(\exists k: e . V C[k]<f . V C[k])))
$$

Let $P_{i}$ be the process that has produced $e$. This additional information allows to simplify the previous relation that reduces to [1, 4]:

$$
(e \rightarrow f) \Leftrightarrow(e . V C[i] \leq f . V C[i]) \quad(R)
$$

Let $\sigma 1$ and $\sigma 2$ be local states of $P_{i}$ and $P_{j}$, respectively. From the definition $S E$ and the previous relations we get: $(\sigma 1 \rightarrow \sigma 2) \Leftrightarrow(\sigma 1 . V C[i]<\sigma 2 . V C[i])$.

Timestamps of Global States Relations between vector clocks and global states have been studied from a formal point of view by several authors [2 4]. Here, we only consider some of these properties through a set of simple examples taken from Figure 1

${ }^{1}(\forall k: e . V C[k] \leq f . V C[k]) \wedge(\exists k:$ e.VC $[k]<f . V C[k])$ is denoted $e . V C<f . V C$. 
Vector clocks allow to associate timestamps to global states in a very easy way. First, let us consider a local state, e.g., $\sigma_{3}^{4}$. It is timestamped $(0,4,4)$ and this timestamp identifies the consistent global state $\left(\sigma_{1}^{0}, \sigma_{2}^{4}, \sigma_{3}^{4}\right)$ which is the first consistent global state to which $\sigma_{3}^{4}$ belongs. More generally, the timestamp of a consistent global state $\Sigma$ is defined as the component-wise maximum of the timestamps of the local states that compose it. Let us consider $\Sigma=\left(\sigma_{1}^{4}, \sigma_{2}^{4}, \sigma_{3}^{4}\right)$. Its timestamp is the component-wise maximum (denoted max) of $(4,3,2),(0,4,2)$ and $(0,4,4)$, i.e., $(4,4,4)$.

In the same way if we consider two (or more) consistent global states $\Sigma_{1}$ and $\Sigma_{2}$, timestamped $\Sigma_{1} \cdot V C$ and $\Sigma_{2} . V C$, respectively, the global state defined by the timestamp $\max \left(\Sigma_{1} . V C, \Sigma_{2} . V C\right)$ is the first consistent global state (called $\left.\max \left(\Sigma_{1}, \Sigma_{2}\right)\right)$ that includes the causal past of both $\Sigma_{1}$ and $\Sigma_{2}$. As an example, let us consider $\Sigma_{1}=\left(\sigma_{1}^{0}, \sigma_{2}^{2}, \sigma_{3}^{3}\right)$ timestamped $(0,2,3)$, and the global state $\Sigma_{2}=\left(\sigma_{1}^{4}, \sigma_{2}^{4}, \sigma_{3}^{2}\right)$ timestamped $(4,4,2)$. The global state $\Sigma=\max \left(\Sigma_{1}, \Sigma_{2}\right)$ is a consistent global state, namely $\left(\sigma_{1}^{4}, \sigma_{2}^{4}, \sigma_{3}^{3}\right)$, timestamped $(4,4,3)$, that occurs after $\Sigma_{1}$ and after $\Sigma_{2}$. (Actually, from a theoretic point of view, the set of consistent global states defines a lattice [2, 4, 6]).

\section{Detection of a Conjunction of Stable Local Predicates}

\subsection{The Problem}

A predicate is local to a process $P_{i}$ if it is only on local variables of $P_{i}$. A local predicate $L P_{i}$ is stable if, as soon as it becomes true, it remains true forever. The notation $\sigma_{i}=L P_{i}$ will be used to indicate that $P_{i}$ 's local state $\sigma_{i}$ satisfies the local predicate $L P_{i}$. Let $L P_{1}, L P_{2}, \ldots, L P_{n}$ be $n$ local predicates, one per process. A consistent global state $\Sigma=\left(\sigma_{1}, \cdots, \sigma_{n}\right)$ satisfies the global predicate $L P_{1} \wedge L P_{2} \cdots \wedge L P_{n}\left(\right.$ denoted $\left.\Sigma \models\left(\bigwedge_{i} L P_{i}\right)\right)$ if $\bigwedge_{i}\left(\sigma_{i} \models L P_{i}\right)$.

The problem consists in detecting on-the-fly, and without using additional control messages, the first consistent global state $\Sigma$ that satisfies a conjunction of stable local predicates.

\subsection{How to Solve It}

Let us consider Figures 2] a and 2]. The fact that local predicate $L P_{i}$ is satisfied, is indicated by thickening process $P_{i}$ 's axis. In both figures, the global state $\Sigma=\left(\sigma_{1}^{y_{1}}, \sigma_{2}^{y_{2}}, \sigma_{3}^{y_{3}}\right)$ (timestamped $\left.\left(y_{1}, y_{2}, y_{3}\right)\right)$ is the first to satisfy the conjunction of stable local predicates. Let us first examine Figure 2 a.

- When $P_{1}$ receives $m_{1}$, it learns nothing new about predicate detection.

- When $P_{1}$ receives $m_{3}$ (i.e., when it enters local state $\sigma_{1}^{x_{1}}$ ), it can learn (by appropriately tracking causality with vector clocks) that there is a consistent global state, namely $\left(\sigma_{1}^{0}, \sigma_{2}^{x_{2}}, \sigma_{3}^{0}\right)$, that partially satisfies the global predicate $\left(\sigma_{2}^{x_{2}} \models L P_{2}\right)$.

- Message $m_{4}$ gives $P_{2}$ the knowledge that $\sigma_{3}^{x_{3}} \models L P_{3}$. So, (using vector clocks) $m_{5}$ can carry the information that the global state timestamped $\left(0, x_{2}, x_{3}\right)$ partially satisfies the global predicate (namely, $\left.\left(\sigma_{1}^{0}, \sigma_{2}^{x_{2}}, \sigma_{3}^{x_{3}}\right) \models L P_{2} \wedge L P_{3}\right)$. 


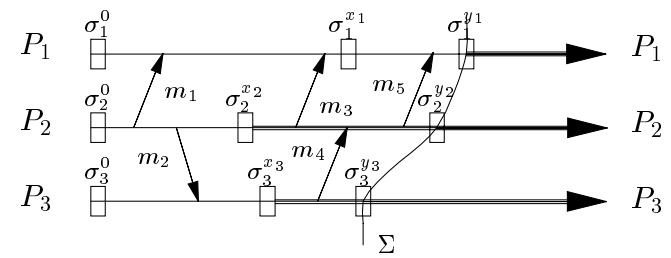

a. Example 1

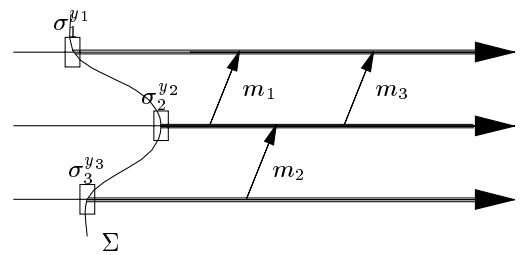

b. Example 2

Fig. 2. Detection of $\bigwedge_{i} L P_{i}$

- So, when $P_{1}$ receives $m_{5}$ it learns this, and when $L P_{1}$ becomes true, $P_{1}$ can safely detects that $\Sigma$, timestamped $\left(y_{1}, y_{2}, y_{3}\right)$, is the first consistent global state satisfying $\bigwedge_{i} L P_{i}\left(\sigma_{2}^{y_{2}}\right.$ and $\sigma_{3}^{y_{3}}$ being the local states that immediately follow the sendings of $m_{5}$ and $m_{4}$, respectively).

Let us now examine Figure 2 $\mathrm{b}$. In that situation, due to flow of exchanged messages, $P_{1}$ can learn, when it receives $m_{3}$, that $\Sigma$, timestamped $\left(y_{1}, y_{2}, y_{3}\right)$, is the first consistent global state satisfying the global predicate $L P_{1} \wedge L P_{2} \wedge L P_{3}$.

\subsection{Data Structures}

The previous discussion shows that we need two thinks to solve the problem: (1) Track causality to be able to identify consistent global states; and (2) Track which local predicates are satisfied and track the first consistent global state in which those local predicates are satisfied. Hence, each process $P_{i}$ is endowed with the following data structures:

- $V C_{i}[1 . . n]$ : a vector clock (initialized to $\left.(0,0, \ldots, 0)\right)$.

- $S A T_{i}$ : a set (initially empty) of process identifiers, with the following meaning: $j \in S A T_{i} \Leftrightarrow P_{i}$ knows $P_{j}$ entered a local state from which $L P_{j}$ is true.

- FIRST $T_{i}$ : a vector timestamp (initialized to $(0,0, \ldots, 0)$ ) that defines the first consistent global state, known by $P_{i}$, in which all the predicates defined in $S A T_{i}$ are satisfied. More precisely: $\forall j:\left(j \in S A T_{i} \Rightarrow\left(\sigma_{j}^{F I R S T_{i}[j]} \models L P_{j}\right)\right)$. Note that if $S A T_{i}=\{1,2, \cdots, n\}$, then $F I R S T_{i}$ defines the first consistent global state $\Sigma$ that satisfies $\bigwedge_{j} L P_{j}$. In Figure $2 \mathrm{a}, F I R S T_{1}=V C_{1}=\left(y_{1}, y_{2}, y_{3}\right)$ when $L P_{1}$ becomes true. In Figure 2, b, when $P_{1}$ receives $m_{3}$, it updates $S A T_{1}$ (the value of which becomes $\{1,2,3\})$ and $F I R S T_{1}$ takes the value $\max \left(\left(y_{1}, y_{2}, 0\right),\left(0, y_{2}, y_{3}\right)\right)$, i.e., FIRST $T_{1}=\left(y_{1}, y_{2}, y_{3}\right)$. In both figures, $\left(\sigma_{1}^{y_{1}}, \sigma_{2}^{y_{2}}, \sigma_{3}^{y_{3}}\right) \models\left(L P_{1} \wedge L P_{2} \wedge L P_{3}\right)$.

\subsection{The Detection Protocol}

The protocol executed by a process $P_{i}$ is described in Figure 3. Let $e$ and $\sigma$ denote the last event produced by $P_{i}$ and the corresponding local state (entered by $P_{i}$ just after executing $e$ ). Their vector timestamps $(e . V C$ and $\sigma . V C)$ have the same value which is equal the current value of $V C_{i}$. The protocol 
is composed of two procedures and three statements $S 1, S 2$ and $S 3$ associated with the production by $P_{i}$ of an internal event, a send event and a receive event, respectively. The local variable done $_{i}$ (initialized to false) is set to the value true the first time $L P_{i}$ is satisfied (then it remains true forever). The notation $V C:=\max (V C 1, V C 2$ ) (statement $\mathrm{S} 3$ ) is an abbreviation for $\forall k \in 1 . . n: V C[k]:=\max (V C 1[k], V C 2[k])$. It defines a vector clock update. The protocol consists in:

procedure detected? is

if $S A T_{i}=\{1,2, \ldots, n\}$ then $F I R S T_{i}$ defines the first consistent

procedure $c h e c k_{-} L P_{i}$ is global state $\Sigma$ that satisfies $\bigwedge_{j} L P_{j}$ fi

if $\left(\sigma_{i}^{x}=L P_{i}\right)$ then $S A T_{i}:=S A T_{i} \cup\{i\} ; F I R S T_{i}:=V C_{i}$;

done $_{i}:=$ true; detected? $\mathbf{f i}$

(S1) when $P_{i}$ produces an internal event $(e)$

$V C_{i}[i]:=V C_{i}[i]+1$; execute $e$ and move to $\sigma$;

if $\neg d_{\text {one }}$ then check_$_{-} L P_{i} \mathbf{f i}$

(S2) when $P_{i}$ produces a send event ( $e=$ send $m$ to $\left.P_{j}\right)$

$V C_{i}[i]:=V C_{i}[i]+1$; move to $\sigma ;$ if $\neg$ done $_{i}$ then check $_{-} L P_{i} \mathbf{f i}$;

$m . V C:=V C_{i} ; m . S A T:=S A T_{i} ;$ m.FIRST $:=F I R S T_{i}$;

send $(m)$ to $P_{j} \% m$ carries m.VC, m.SAT and m.FIRST \%

(S3) when $P_{i}$ produces a receive event $(e=$ receive $(m))$

$V C_{i}[i]:=V C_{i}[i]+1 ; V C_{i}:=\max \left(V C_{i}, m . V C\right)$;

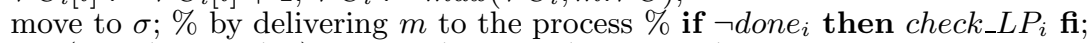
if $\neg\left(m . S A T \subseteq S A T_{i}\right)$ then $S A T_{i}:=S A T_{i} \cup m . S A T$;

$F I R S T_{i}:=\max \left(F I R S T_{i}\right.$, m.FIRST);

detected? fi

Fig. 3. Detection Protocol for $\bigwedge_{i} L P_{i}$

- The management of the vector clock $V C_{i}$ (in $S 1, S 2$ and $S 3$ ).

- The combined management of variables $S A T_{i}$ and $F I R S T_{i}$ :

- When $L P_{i}$ becomes true, $V C_{i}$ defines the first consistent global state in which $L P_{i}$, plus the local predicates already in $S A T_{i}$, are satisfied. So $F I R S T_{i}$ is defined as $V C_{i}$ (procedure check_L $L P_{i}$ ).

- When $P_{i}$ sends a message $m$ (Statement $S 2$ ), it attaches to it the current values of $S A T_{i}$ and FIRST . Those values are denoted m.SAT and m.FIRST.

- When $P_{i}$ receives a message $m$ (Statement S3), it first executes the reception, moves to the new current state and tests if its local predicate becomes satisfied. Then, $P_{i}$ checks if it learns something new about the satisfaction of local local predicates (by testing $\neg\left(m . S A T \subseteq S A T_{i}\right)$ ). If it learns something new, it computes the first consistent global state in which the local predicates of m.SAT $\cup S A T_{i}$ are satisfied. As indicated in the previous discussion, this global state is timestamped $\max \left(F I R S T_{i}, m . F I R S T\right)$. 
- When something new happens (from the point of view of local predicates detection), $P_{i}$ tests the condition $S A T_{i}=\{1,2, \cdots, n\}$, to know if the full global predicate, namely $\bigwedge_{i} L P_{i}$, is satisfied.

\section{Limitation of Simple Vector Clocks}

Vector clocks provide each process with a "counter-based view" of its causal past that displays limitations to solve some "causality"-related problems. We present here such a problem and its solution.

\subsection{Recognition of a Simple Pattern}

Let us consider a distributed execution that produces two types of internal events: black and white (colmmunication events are tagged white). Given two blacks events $s$ and $t$, the problem consists in deciding if there is another black event $u$ such that $s \rightarrow u \wedge u \rightarrow t$ ? Let black(e) be a predicate indicating if event $e$ is black. More formally, given two events $s$ and $t$, the problem consists in deciding if the following predicate $\mathcal{P}(s, t)$ is true:

$$
\mathcal{P}(s, t) \equiv(\operatorname{black}(s) \wedge \operatorname{black}(t)) \wedge(\exists u \neq s, t:(\operatorname{black}(u) \wedge(s \rightarrow u \wedge u \rightarrow t)))
$$

To show that vector clocks do not allow to solve this problem let us consider Figures 4.a and 4.b. In these two executions, both event $s$ have the same timestamp: $s . V C=(0,0,2)$. Similarly, both events $t$ have also the same timestamp, namely, $t . V C=(3,4,2)$. But, as the reader can verify, execution (b) satisfies the pattern, while (a) does not.

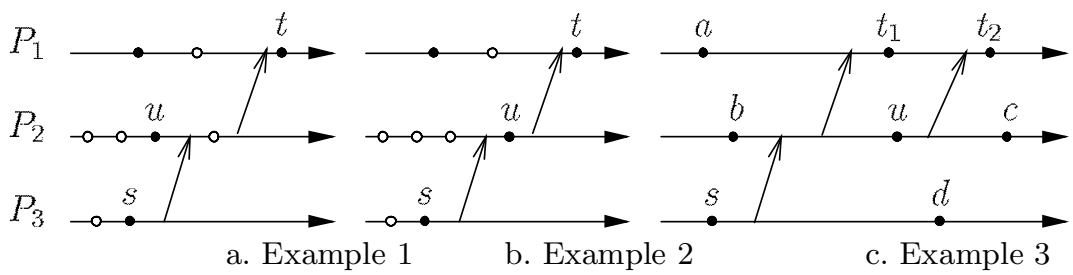

Fig. 4. Recognize a Pattern

\subsection{How to Solve It}

Observe that for the predicate $\mathcal{P}(s, t)$ to be true, there must be a black event in the causal past of $t$ which has $s$ in its causal past. This problem is related to causality, but two levels of predecessors appear in the predicate $\mathcal{P}$. Track "second order knowledge" on the past requires vector of vectors clocks. 
Which Clocks? $\mathcal{P}(s, t)$ can be decomposed into two predicates $\mathcal{P}_{1}(s, u, t)$ and $\mathcal{P}_{2}(s, u, t)$ in the following way: $\mathcal{P}(s, t) \equiv\left(\exists u: \mathcal{P}_{1}(s, u, t) \wedge \mathcal{P}_{2}(s, u, t)\right)$, where $\mathcal{P}_{1}(s, u, t) \equiv(\operatorname{black}(s) \wedge \operatorname{black}(u) \wedge \operatorname{black}(t))$ and $\mathcal{P}_{2}(s, u, t) \equiv(s \rightarrow u \wedge u \rightarrow t)$.

$\mathcal{P}_{1}$ indicates that only the black events are relevant for the predicate detection: so, only black events have to be tracked. This means vector clocks can be managed in the following way: (1) A process $P_{i}$ increments $V C_{i}[i]$ only when it produces a black event; (2) The other statements associated with vector clocks are left unchanged.

Let us consider Figure 4.c, where only black events are indicated. We have $\mathcal{P}\left(s, t_{1}\right)=$ false, while (due to $u$ or to $\left.t_{1}\right) \mathcal{P}\left(s, t_{2}\right)=$ true. The underlying idea to solve the problem lies in associating two timestamps with each black event $e$ : - A vector timestamp e.VC (counting only black events).

- An array of vector timestamps e.MC $[1 . . n]$ such that e.MC $[j]$ contains the vector timestamp of the last black event of $P_{j}$ that causally precedes $e(e . M C[j]$ can be considered as a pointer from $e$ to the last event that precedes it on $P_{j}$ ). When considering Figure 4.c, we have: $t_{1} \cdot M C[1]=a \cdot V C, t_{1} \cdot M C[2]=b . V C$, $t_{1} \cdot M C[3]=s \cdot V C, t_{2} \cdot M C[1]=t_{1} \cdot V C, t_{2} \cdot M C[2]=u . V C$ and $t_{2} \cdot M C[3]=s . V C$.

Each process $P_{i}$ has a vector clock $V C_{i}[1 . . n]$ and a vector of vector clocks $M C_{i}[1 . . n]$. Those variables are managed as described in Figure 5, Let us note that, in statement $\mathrm{S} 3, M C_{i}[k]$ and $m . M C[k]$ contain vector timestamps of two black events of $P_{k}$. It follows that one of them is greater or equal to the other: $\max \left(M C_{i}[k], m . M C[k]\right)$ is its timestamp.

(S1) when $P_{i}$ produces a black event $(e)$

$V C_{i}[i]:=V C_{i}[i]+1 ; \%$ one more black event on $P_{i} \%$ $e . V C=V C_{i} ; e \cdot M C=M C_{i}$;

$M C_{i}[i]:=V C_{i} \%$ vector timestamp of $P_{i}$ 's last black event \%

(S2) when $P_{i}$ executes a send event $\left(e=\right.$ send $m$ to $\left.P_{j}\right)$ $m . V C:=V C_{i} ; m . M C:=M C_{i}$;

send $(m)$ to $P_{j} \% m$ carries $m . V C$ and $m . M C \%$

(S3) when $P_{i}$ executes a receive event $(e=$ receive $(m))$

$V C_{i}:=\max \left(V C_{i}, m . V C\right) ; \%$ update of the local vector clock \%

$\forall k: M C_{i}[k]:=\max \left(M C_{i}[k], m . M C[k]\right)$

$\%$ record vector timestamp of the last black predecessor on each $P_{k} \%$

Fig. 5. Clock Management for Detecting $\mathcal{P}(s, t)$

An Operational Pattern Detection Predicate Let us first remark that, as the protocol considers only black events, the predicate $\mathcal{P}_{1}$ is trivially satisfied by any triple of events. So, detecting $\mathcal{P}(s, t)$ amounts to only detect $\exists u: \mathcal{P}_{2}(s, u, t)$. Given $s$ and $t$ with their timestamps (namely, s.VC and s.MC for $s ; t . V C$ and $t . M C$ for $t)$, the predicate $\left(\exists u: \mathcal{P}_{2}(s, u, t)\right) \equiv(\exists u: s \rightarrow u \rightarrow t)$ can be 
restated in a more operational way using vector timestamps. More precisely: $(\exists u: s \rightarrow u \rightarrow t) \equiv(\exists u: s . V C<u . V C<t . V C)$.

If such an event $u$ does exist, it has been produced by some process $P_{k}$ and belongs to the causal past of $t$. Consequently, its vector timestamp is such that: $\exists k: u . V C \leq t . M C[k]$. From this observation, the previous relation translates in: $(\exists u: s \rightarrow u \rightarrow t) \equiv(\exists k: s . V C<t . M C[k]<t . V C)$.

As $\forall k, t . M C[k]$ is the vector timestamp of a black event in the causal past of $t$, we have $\forall k: t . M C[k]<t . V C$. Consequently, the pattern detection predicate simplifies and becomes: $\mathcal{P}(s, t) \equiv(\exists k: s . V C<t . M C[k])$.

\section{References}

[1] Fidge C.J., Timestamp in Message Passing Systems that Preserves Partial Ordering, Proc. 11th Australian Computing Conference, pp. 56-66, 1988.

[2] Garg V.K., Principles of Distributed Systems, Kluwer Acad. Press, 274 pages, 1996.

[3] Lamport L., Time, Clocks and the Ordering of Events in a Distributed System, Communications of the ACM, 21(7):558-565, 1978.

[4] Mattern F., Virtual Time and Global States of Distributed Systems, Proc. "Parallel and Distributed Algorithms" Conference, North-Holland, pp. 215-226, 1988.

[5] Raynal M. and Singhal S., Logical Time: Capturing Causality in Distributed Systems, IEEE Computer, 29(2):49-57, 1996.

[6] Schwarz R. and Mattern F., Detecting Causal Relationships in Distributed Computations: in Search of the Holy Grail. Distributed Computing, 7:149-174, 1994. 